\title{
An Efficient Diagnosis System for Parkinson's Disease Using Kernel-Based Extreme Learning Machine with Subtractive Clustering Features Weighting Approach
}

\author{
Chao Ma, ${ }^{1,2}$ Jihong Ouyang, ${ }^{1,2}$ Hui-Ling Chen, ${ }^{3}$ and Xue-Hua Zhao ${ }^{1,2}$ \\ ${ }^{1}$ College of Computer Science and Technology, Jilin University, No. 2699, QianJin Road, Changchun 130012, China \\ ${ }^{2}$ Key Laboratory of Symbolic Computation and Knowledge Engineering of Ministry of Education, Jilin University, \\ Changchun 130012, China \\ ${ }^{3}$ College of Physics and Electronic Information, Wenzhou University, Wenzhou 325035, China
}

Correspondence should be addressed to Jihong Ouyang; 1sw_1017@aliyun.com

Received 21 June 2014; Accepted 26 October 2014; Published 18 November 2014

Academic Editor: Dong Song

Copyright $\odot 2014$ Chao Ma et al. This is an open access article distributed under the Creative Commons Attribution License, which permits unrestricted use, distribution, and reproduction in any medium, provided the original work is properly cited.

\begin{abstract}
A novel hybrid method named SCFW-KELM, which integrates effective subtractive clustering features weighting and a fast classifier kernel-based extreme learning machine (KELM), has been introduced for the diagnosis of PD. In the proposed method, SCFW is used as a data preprocessing tool, which aims at decreasing the variance in features of the PD dataset, in order to further improve the diagnostic accuracy of the KELM classifier. The impact of the type of kernel functions on the performance of KELM has been investigated in detail. The efficiency and effectiveness of the proposed method have been rigorously evaluated against the PD dataset in terms of classification accuracy, sensitivity, specificity, area under the receiver operating characteristic (ROC) curve (AUC), $f$-measure, and kappa statistics value. Experimental results have demonstrated that the proposed SCFW-KELM significantly outperforms SVM-based, KNN-based, and ELM-based approaches and other methods in the literature and achieved highest classification results reported so far via 10-fold cross validation scheme, with the classification accuracy of $99.49 \%$, the sensitivity of $100 \%$, the specificity of $99.39 \%$, AUC of $99.69 \%$, the $f$-measure value of 0.9964 , and kappa value of 0.9867 . Promisingly, the proposed method might serve as a new candidate of powerful methods for the diagnosis of PD with excellent performance.
\end{abstract}

\section{Introduction}

Parkinson's disease (PD) is one degenerative disease of the nervous system, which is characterized by a large group of neurological conditions called motor system disorders because of the loss of dopamine-producing brain cells. The main symptoms of PD are given as follows: (1) tremor or trembling in hands, arms, legs, jaw, or head, (2) rigidity or stiffness of the limbs and trunk, (3) bradykinesia or slowness of movement, (4) postural instability or impaired balance (http://www.ninds.nih.gov/research/parkinsonsweb/index. htm, last accessed: April 2012). At present, PD has an impact on about $1 \%$ of the worldwide population over the age of 50; however, this proportion is on the increase as people live longer [1]. Till now, PD has no medical treatment and some dedication is only available for relieving the symptoms of disease [2]. It is so important that we gain more of insight into the problem and improve our methods to deal with PD. Here we focus on the study based on dysphonia, which is known as a group of vocal impairment symptoms; it is reported to be one of the most significant symptoms of PD [3]. The researches have shown that about $90 \%$ of people with PD have such vocal evidence. The dysphonic indicators of PD make speech measurements as an important part of diagnosis [4]. Dysphonic measures have been proposed as a reliable tool to detect and monitor PD $[5,6]$.

Previous studies on the PD problem based on machine learning methods have been undertaken by various researchers. Little et al. [6] used support vector machine (SVM) classifier with Gaussian radical basis kernel function to predict $\mathrm{PD}$, by means of feature selection method to reduce the feature space, and best accuracy rate of $91.4 \%$ was 
obtained by the proposed model. Shahbaba and Neal [7] presented a nonlinear model based on Dirichlet mixtures for the PD classification, compared with multinomial logit models, decision trees, and SVM; the classification accuracy of $87.7 \%$ was achieved by the proposed model. Das [8] used a comparative study of neural networks (NN), DMneural, regression and decision trees for the diagnosis of $\mathrm{PD}$; the experiment results had shown that the NN method achieved the overall classification performance of $92.9 \%$. Sakar and Kursun [9] used mutual information measure to combine with SVM for the diagnosis of PD and achieved the classification result of $92.75 \%$. Psorakis et al. [10] introduced sample selection strategies and model improvements for multiclass multikernel relevance vector machines and achieved the classification accuracy of $89.47 \%$ in the PD dataset. Guo et al. [11] combined genetic programming and the expectation maximization (EM) to diagnose PD in the ordinary feature data and achieved the classification accuracy of $93.1 \%$. Luukka [12] proposed a new method which used fuzzy entropy measures to combine with the similarity classifier to predict $\mathrm{PD}$, and the mean classification of 85.03\% was achieved. Li et al. [13] introduced a fuzzy-based nonlinear transformation approach together with SVM in the PD dataset; best classification accuracy of $93.47 \%$ was obtained. Ozcift and Gulten [14] combined the correlation based feature selection method with the rotation forest ensemble classifier of 30 machine learning algorithms to distinguish PD; the proposed model got best classification accuracy of $87.13 \%$. Åström and Koker [15] achieved highest classification accuracy of $91.2 \%$ by using a parallel neural network model for PD diagnosis. Spadoto et al. [16] adopted evolutionary based method together with the optimum-path forest (OPF) classifier for PD diagnosis, and best classification accuracy of $84.01 \%$ was obtained. Polat [17] applied the fuzzy $C$-means (FCM) clustering feature weighting (FCMFW) together with the $k$-nearest neighbor classifier for detecting PD; the classification accuracy of $97.93 \%$ was obtained. Chen et al. [18] proposed a model which used the principle component analysis based feature extraction together with the fuzzy $k$-nearest neighbor method to predict PD and achieved best classification accuracy of $96.07 \%$ by the proposed model. Daliri [19] presented a chi-square distance kernel-based SVM to discriminate the subjects with PD from the healthy control subjects using gait signals, and the classification result of $91.2 \%$ was obtained. Zuo et al. [20] used a new diagnosis model based on particle swarm optimization (PSO) to strengthen the fuzzy $k$-nearest neighbor classifier for the diagnosis of $\mathrm{PD}$, and the mean classification accuracy of $97.47 \%$ was achieved.

From these works, it can be seen that most of the common classifiers from machine learning community have been used for PD diagnosis. For the nonlinear classification problems, the data preprocessing methods such as feature weighting, normalization, and feature transformation could increase the performance of alone classifier algorithm. So it is obvious that the choice of an efficient feature preprocessing method and an excellent classifier is of significant importance for the PD diagnosis problem. Aiming at improving the efficiency and effectiveness of the classification performance for the diagnosis of PD, in this paper, an efficient features weighting method called subtractive clustering features weighting (SCFW) and a fast classification algorithm named kernelbased extreme learning machine (KELM) are examined. The SCFW method is used to map the features according to data distributions in dataset and transform linearly nonseparable dataset to linearly separable dataset. In this way, the similar data within each feature are prone to getting together so that the distinction between classes is increased to classify the PD datasets correctly. It is reported that SCFW method can help improve the discrimination abilities of classifiers in many applications, such as traffic accident analysis [21] and medical datasets transformation [22]. KELM is the improved version of ELM algorithm based on kernel function [23]. The advantage of KELM is that only two parameters (the penalty parameter $C$ and the kernel parameter $\gamma$ ) need to be adjusted, unlike ELM which needs to specify the suitable values of weights and biases for improving the generalization performance [24]. Furthermore, KELM not only trains as fast as that of ELM, but also can achieve good generalization performance. The objective of the proposed method is to explore the performance of PD diagnosis using a two-stage hybrid modeling procedure via integrating SCFW with KELM. Firstly the proposed method adopts SCFW to construct the discriminative feature space through weighting features, and then the achieved weighted features serve as the input of the trained KELM classifier. To evaluate the performance of proposed hybrid method, classification accuracy (ACC), sensitivity, specificity, AUC, $f$-measure, and kappa statistic value have been used. Experimental results have shown that the proposed method achieves very promising results based on proper kernel function by 10 -fold cross validation (CV).

The main contributions of this paper are summarized as follows.

(1) It is the first time that we have proposed to integrate SCFW approach with KELM classifier to detect PD in an efficient and effective way.

(2) In the proposed system, SCFW method is employed as data preprocessing tool to strengthen the discrimination between classes for further improving the distinguishing performance of KELM classifier.

(3) Compared with the existing methods in previous studies, the proposed diagnostic system has achieved excellent classification results.

The rest of the paper is organized as follows. Section 2 offers brief background knowledge on SCFW and KELM. The detailed implementations of the diagnosis system are presented in Section 3. In the next section, the detailed experiment design is described, and Section 5 gives the experiment results and discussions of the proposed method. Finally, conclusions and recommendations for future work are summarized in Section 6. 


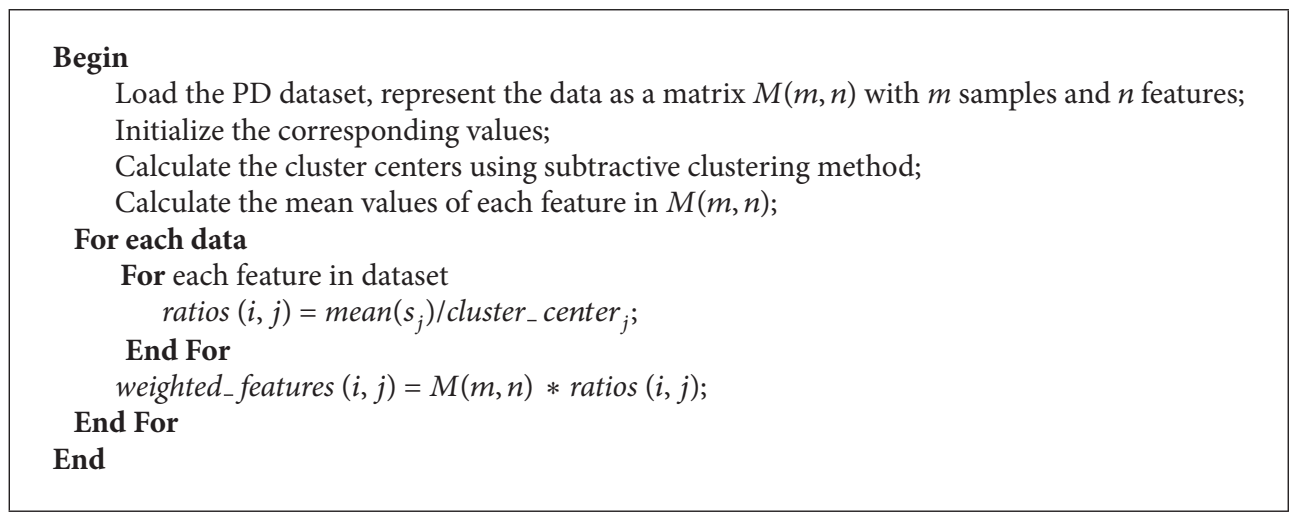

Algorithm 1: Pseudocode for weighting features based on subtractive clustering method.

\section{The Theoretical Background of the Related Methods}

2.1. Subtractive Clustering Features Weighting (SCFW). Subtractive clustering is the improved version of mountain clustering algorithm. The problem of mountain clustering is that its calculation grows exponentially with the dimension of the problem. Subtractive clustering has solved this problem using data points as the candidates for cluster centers, instead of grid points as in mountain clustering, so the calculation cost is proportional to the problem size instead of the problem dimension [25]. The subtractive clustering algorithm can be briefly summarized as follows:

Step 1. Consider a collection of $n$ data points $\left\{x_{1}, x_{2}, \ldots, x_{n}\right\}$ in $M$-dimensional space. Since each data point is a candidate for cluster center, the density measure at data point $x_{i}$ is defined as

$$
D_{i}=\sum_{j=1}^{n} \exp \left(\frac{-\left\|x_{i}-x_{j}\right\|^{2}}{\left(r_{a} / 2\right)^{2}}\right),
$$

where $r_{a}$ is a positive constant defining a neighborhood radius; it is used to determine the number of cluster centers. So, a data point will have a high density value if it has many neighboring data points. The data points outside the neighborhood radius contribute slightly to the density measure. Here, $r_{a}$ is set to 0.5 .

Step 2. After the density measure of each data point has been calculated, the data point with the highest density measure is selected as the first cluster center. Let $X_{c 1}$ be the point selected and $D_{c 1}$ the density measure. Next, the density measure for each data point $x_{i}$ is revised as follows:

$$
D_{i}=D_{i}-D_{c 1} \exp \left(\frac{-\left\|x_{i}-x_{j}\right\|^{2}}{\left(r_{b} / 2\right)^{2}}\right) \text {, }
$$

where $r_{b}$ is a positive constant and $r_{b}=\eta \cdot r_{a}, \eta$ is a constant greater than 1 to avoid cluster centers being in too close proximity. In this paper, $r_{b}$ is set to 0.8 .

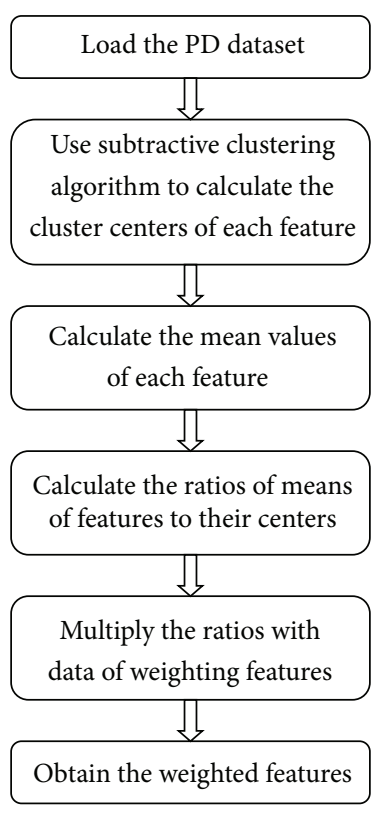

FIGURE 1: The flowchart of SCFW algorithm.

Step 3. After the density calculation for each data point is revised, the next cluster center $X_{c 2}$ is selected and all the density calculations for data point are revised again. The process is repeated until a sufficient number of cluster centers are generated.

For SCFW method, firstly the cluster centers of each feature are calculated by using subtractive clustering. After calculating the centers of features, the ratios of means of features to their cluster centers are calculated and these ratios are multiplied with the data of each feature [21]. The pseudocode of SCFW method is given in Algorithm 1, and the flowchart of weighting process is shown in Figure 1.

2.2. Kernel-Based Extreme Learning Machine (KELM). ELM is an algorithm originally developed for training single hidden layer feed-forward neural networks (SLFNs) [26]. The essence of ELM is that parameters of hidden neurons in neural network are randomly created instead of being tuned 


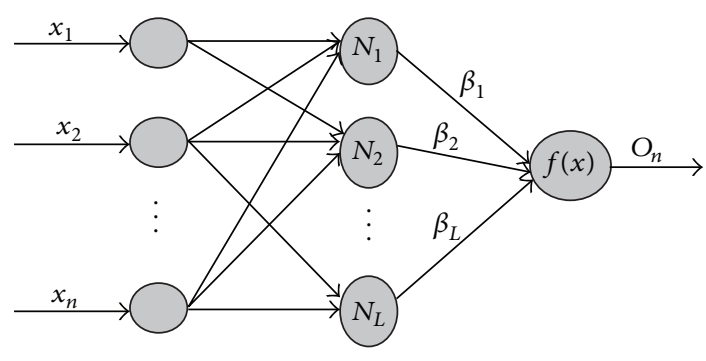

FIgURE 2: The structure of ELM.

and then fixed the nonlinearities of the network without iteration. Figure 2 shows the structure of ELM.

For given $N$ samples $(\mathbf{x}, \mathbf{y})$ having $L$ hidden neurons and activation function $h(x)$, the output function of ELM is defined as follows:

$$
f(x)=\sum_{i=1}^{L} \beta_{i} h_{i}(x)=h(x) \beta,
$$

where $\boldsymbol{\beta}=\left[\beta_{1}, \beta_{2}, \ldots, \beta_{L}\right]$ is the output weight connecting hidden nodes to output nodes. $\mathbf{H}=\left\{h_{i j}\right\}(i=$ $1, \ldots, N$ and $j=1, \ldots, L)$ is the hidden layer output matrix of neural network. $h(x)$ actually maps the data from the ddimensional input space to the $\mathbf{L}$-dimensional hidden layer feature space $\mathbf{H}$, and thus, $h(x)$ is indeed a feature mapping.

The determination of the output weights is calculated by the least square method:

$$
\boldsymbol{\beta}^{\prime}=\mathbf{H}^{+} \mathbf{T},
$$

where $\mathbf{H}^{+}$is the Moore-Penrose generalized inverse [26] of the hidden layer output matrix $\mathbf{H}$.

To improve the generalization capabilities of ELM in comparison with the least square solution-based ELM, Huang et al. [23] proposed kernel-based method for the design of ELM. They suggested adding a positive value $1 / C$ (where $C$ is a user-defined parameter) for calculating the output weights such that

$$
\beta=H^{T}\left(\frac{I}{C}+H H^{T}\right)^{-1} T .
$$

Therefore, the output function is expressed as follows:

$$
f(x)=h \beta=h(x) H^{T}\left(\frac{I}{C}+H H^{T}\right)^{-1} T .
$$

When the hidden feature mapping function $h(x)$ is unknown, a kernel matrix for ELM is used according to the following equation:

$$
\Omega_{\mathrm{ELM}}=H H^{T}: \Omega_{\mathrm{ELM} i, j}=h\left(x_{i}\right) \cdot h\left(x_{j}\right)=K\left(x_{i}, x_{j}\right),
$$

where $K\left(x_{i}, x_{j}\right)$ is a kernel function. Many kernel functions, such as linear, polynomial, and radial basis function, can be used in kernel-based ELM. Now the output function of KELM classifier can be expressed as

$$
f(x)=\left[\begin{array}{c}
K\left(x, x_{1}\right) \\
\vdots \\
K\left(x, x_{N}\right)
\end{array}\right]^{T}\left(\frac{I}{C}+\Omega_{\mathrm{ELM}}\right)^{-1} T .
$$

\section{The Proposed SCFW-KELM Diagnosis System}

This work proposes a novel hybrid method for PD diagnosis. The proposed model is comprised of two stages as shown in Figure 3. In the first stage, SCFW algorithm is firstly applied to preprocess data in the PD dataset. The purpose of this method is to map the features according to their distributions in dataset and to transform from linearly nonseparable space to linearly separable one. With this method, similar data in the same feature are gathered, which will substantially help improve the discrimination ability of classifiers. In the next stage, KELM is evaluated on the weighted feature space with different types of activation functions to perform the classification. Finally, the best parameters and the suitable activation function are obtained based on the performance analysis. The detailed pseudocode of the hybrid method is given in Algorithm 2.

\section{Experimental Design}

4.1. Data Description. In this section, we have performed the experiments in the PD dataset taken from University of California Irvine (UCI) machine learning repository (http:// archive.ics.uci.edu/ml/datasets/Parkinson, last accessed: January 2013). It was created by Max Little of the University of Oxford, in collaboration with the National Centre for Voice and Speech, Denver, Colorado, who recorded the speech signals. The purpose of PD dataset is to discriminate healthy people from those with $\mathrm{PD}$, given the results of various medical tests carried out on a patient. The PD dataset consists of voice measurements from 31 people of which 23 were diagnosed with PD. There are 195 instances comprising 48 healthy and $147 \mathrm{PD}$ cases in the dataset. The time since diagnoses ranged from 0 to 28 years, and the ages of the subjects ranged from 46 to 85 years (mean 65.8). Each subject provides an average of six phonations of the vowel (yielding 195 samples in total), each 36 seconds in length [6]. Note that there are no missing values in the dataset and the whole features are real value. The whole 22 features along with description are listed in Table 1.

4.2. Experimental Setup. The proposed SCFW-KELM classification model was carried out on the platform of MATLAB 7.0. The SCFW algorithm was implemented from scratch. For KELM and ELM, the implementation available from http://www3.ntu.edu.sg/home/egbhuang/ was used.

For SVM, LIBSVM implementation was used, which was originally developed by Chang and Lin [27]. The empirical experiment was conducted on Intel Dual-Core TM $(2.0 \mathrm{GHz}$ CPU) with $2 \mathrm{~GB}$ of RAM.

In order to guarantee the valid results, $k$-fold $C V$ was used to evaluate the classification results [28]. Each time, nine of ten subsets were put together to form a training set and the other subset was used as the test set. Then the average result across all 10 trials was calculated. Thanks to this method, all the test sets were independent and the reliability of the results 


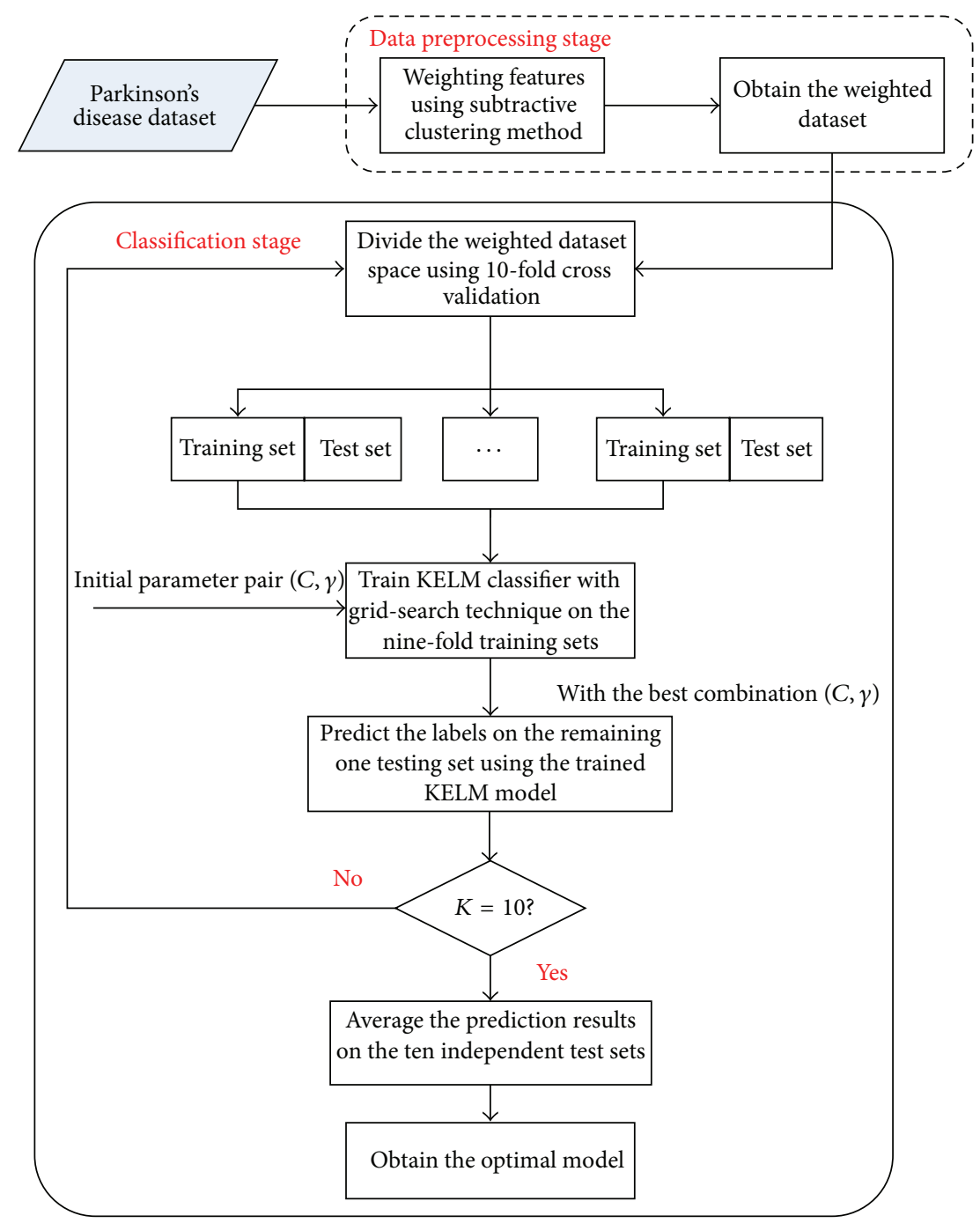

FIGURE 3: The overall procedure of the proposed hybrid diagnosis system.

\section{Begin}

Weight features using subtractive clustering algorithm;

For $i=1: k /^{*}$ Performance estimation by using $k$-fold CV, where $k=10^{*} /$

Training set $=k-1$ subsets;

Test set $=$ remaining subset;

Train KELM classifier in the weighted training data feature space, store the best parameter combination;

Test the trained KELM model on the test set using the achieved best parameter combination;

End For

Return the average classification results of KELM over $i$ th test set;

End

Algorithm 2: Pseudocode for the proposed model.

could be improved. Because of the arbitrariness of partition of the dataset, the predicted results of model at each iteration were not necessarily the same. To evaluate accurately the performance of the PD dataset, the experiment was repeated 10 times and then the results were averaged.
4.3. Measure for Performance Evaluation. In order to evaluate the prediction performance of SCFW-KELM model, we used six performance metrics, ACC, sensitivity, specificity, AUC, $f$-measure, and kappa statistic value, to test the performance of the proposed model. About the mentioned performance 
TABLE 1: The details of the whole 22 features of the PD dataset.

\begin{tabular}{|c|c|c|}
\hline Label & Feature & Description \\
\hline $\mathrm{F} 1$ & MDVP: Fo (Hz) & $\begin{array}{l}\text { Average vocal } \\
\text { fundamental frequency }\end{array}$ \\
\hline $\mathrm{F} 2$ & MDVP: Fhi (Hz) & $\begin{array}{l}\text { Maximum vocal } \\
\text { fundamental frequency }\end{array}$ \\
\hline F3 & MDVP: Flo (Hz) & $\begin{array}{l}\text { Minimum vocal } \\
\text { fundamental frequency }\end{array}$ \\
\hline $\mathrm{F} 4$ & MDVP: Jitter (\%) & $\begin{array}{l}\text { Several measures of } \\
\text { variation in } \\
\text { fundamental frequency }\end{array}$ \\
\hline F5 & $\begin{array}{l}\text { MDVP: Jitter } \\
\quad(\text { Abs })\end{array}$ & \\
\hline F6 & MDVP: RAP & \\
\hline F7 & MDVP: PPQ & \\
\hline F8 & Jitter: PPQ & \\
\hline F9 & MDVP: Shimmer & $\begin{array}{l}\text { Several measures of } \\
\text { variation in amplitude }\end{array}$ \\
\hline F10 & $\begin{array}{l}\text { MDVP: Shimmer } \\
\text { (dB) }\end{array}$ & \\
\hline F11 & Shimmer: APQ3 & \\
\hline F12 & Shimmer: APQ5 & \\
\hline F13 & MDVP: APQ & \\
\hline F14 & Shimmer: DDA & \\
\hline F15 & NHR & $\begin{array}{l}\text { Two measures of ratio } \\
\text { of noise to tonal } \\
\text { components in the } \\
\text { voice }\end{array}$ \\
\hline F16 & HNR & \\
\hline F17 & RPDE & $\begin{array}{l}\text { Two nonlinear } \\
\text { dynamical complexity } \\
\text { measures }\end{array}$ \\
\hline F18 & $\mathrm{D} 2$ & \\
\hline F19 & DFA & $\begin{array}{l}\text { Signal fractal scaling } \\
\text { exponent }\end{array}$ \\
\hline F20 & Spread1 & $\begin{array}{l}\text { Three nonlinear } \\
\text { measures of } \\
\text { fundamental frequency } \\
\text { variation }\end{array}$ \\
\hline F21 & Spread2 & \\
\hline F22 & PPE & \\
\hline
\end{tabular}

evaluation formulations are defined as follows according to the confusion matrix which is shown in Table 2 :

$$
\begin{gathered}
\mathrm{ACC}=\frac{\mathrm{TP}+\mathrm{TN}}{\mathrm{TP}+\mathrm{FP}+\mathrm{FN}+\mathrm{TN}} \times 100 \%, \\
\text { Sensitivity }=\frac{\mathrm{TP}}{\mathrm{TP}+\mathrm{FN}} \times 100 \%, \\
\text { Specificity }=\frac{\mathrm{TN}}{\mathrm{FP}+\mathrm{TN}} \times 100 \%, \\
\text { Precision }=\frac{\mathrm{TP}}{\mathrm{TP}+\mathrm{FP}},
\end{gathered}
$$

\begin{tabular}{|c|c|c|}
\hline & $\begin{array}{c}\text { Predicted patients } \\
\text { with PD }\end{array}$ & $\begin{array}{c}\text { Predicted healthy } \\
\text { persons }\end{array}$ \\
\hline $\begin{array}{l}\text { Actual patients } \\
\text { with PD }\end{array}$ & True positive (TP) & False negative (FN) \\
\hline $\begin{array}{l}\text { Actual healthy } \\
\text { persons }\end{array}$ & False positive (FP) & True negative (TN) \\
\hline \multicolumn{3}{|c|}{ Recall $=\frac{T P}{T P+F}$} \\
\hline
\end{tabular}

TABLE 2: The confusion matrix.

In the confusion matrix, $\mathrm{TP}$ is the number of true positives, which represents that some cases with PD class are correctly classified as PD. FN is the number of false negatives, which represents that some cases with the PD class are classified as healthy. TN is the number of true negatives, which represents that some cases with the healthy class are correctly classified as healthy and FP is the number of false positives, which represents that some cases with the healthy class are classified as PD. ACC is a widely used metric to determine class discrimination ability of classifiers. The receiver operating characteristic (ROC) curve is usually plotted using true positives rate versus false positives rate, as the discrimination threshold of classification algorithm is varied. The area under ROC curve (AUC) is widely used in classification studies with relevant acceptance and it is a good summary of the performance of the classifier [29]. Also $f$-measure is a measure of a test's accuracy, which is usually used as performance evaluation metric to assess the performance of binary classifier, based on the harmonic mean for the classifier's precision and recall. Kappa error (KE) or Cohen's kappa statistics (KS) is adopted to compare the performances of different classifiers. KS is a good measure to inspect classifications that may be due to chance. As KS value calculated for classifiers closer to 1 , the performance of classifier is assumed to be more realistic rather than being by chance. Thus, KS value is a recommended metric to consider for evaluation in the performance analysis of classifiers and it is calculated with [30]

$$
\mathrm{KS}=\frac{P(A)-P(E)}{1-P(E)},
$$

where $P(A)$ means total agreement probability and $P(E)$ means agreement probability due to chance.

\section{Experimental Results and Discussions}

Experiment 1 (classification in the $\mathrm{PD}$ dataset). In this experiment, we firstly evaluated KELM in the original feature space without SCFW. It is known that different types of kernel activation functions have great influence on the performance of KELM. Therefore, we presented the results from our investigation on the influence of different types of 
TABLE 3: Results of KELM with different types of kernel functions in the original PD dataset without SCFW.

\begin{tabular}{|c|c|c|c|c|c|}
\hline Kernel type & $\begin{array}{l}\text { Performance } \\
\text { metrics }\end{array}$ & Mean & SD & Max & Min \\
\hline \multirow{6}{*}{ RBF_kernel } & $\operatorname{ACC}(\%)$ & 95.89 & 4.66 & 100 & 89.74 \\
\hline & Sensitivity (\%) & 96.35 & 5.19 & 100 & 88.89 \\
\hline & Specificity (\%) & 95.72 & 5.93 & 100 & 88.00 \\
\hline & AUC (\%) & 96.04 & 4.06 & 100 & 90.43 \\
\hline & $f$-measure & \multicolumn{4}{|c|}{0.9724} \\
\hline & Kappa & \multicolumn{4}{|c|}{0.8925} \\
\hline \multirow{6}{*}{ Wav_kernel } & $\operatorname{ACC}(\%)$ & 94.36 & 4.59 & 100 & 87.18 \\
\hline & Sensitivity (\%) & 91.24 & 6.02 & 100 & 83.33 \\
\hline & Specificity (\%) & 95.15 & 5.23 & 100 & 86.21 \\
\hline & AUC (\%) & 93.19 & 4.56 & 100 & 88.10 \\
\hline & $f$-measure & \multicolumn{4}{|c|}{0.9622} \\
\hline & Kappa & \multicolumn{4}{|c|}{0.8425} \\
\hline \multirow{6}{*}{ Lin_kernel } & ACC (\%) & 89.23 & 7.99 & 97.44 & 79.49 \\
\hline & Sensitivity (\%) & 66.07 & 22.33 & 90.91 & 41.67 \\
\hline & Specificity (\%) & 97.32 & 2.80 & 100 & 93.33 \\
\hline & AUC (\%) & 81.70 & 12.22 & 95.45 & 68.89 \\
\hline & $f$-measure & \multicolumn{4}{|c|}{0.9316} \\
\hline & kappa & \multicolumn{4}{|c|}{0.6333} \\
\hline \multirow{6}{*}{ Poly_kernel } & ACC (\%) & 90.77 & 4.29 & 97.44 & 87.18 \\
\hline & Sensitivity (\%) & 87.73 & 11.54 & 100 & 75.00 \\
\hline & Specificity (\%) & 91.83 & 5.73 & 96.77 & 82.76 \\
\hline & AUC (\%) & 89.78 & 5.78 & 98.39 & 82.66 \\
\hline & $f$-measure & \multicolumn{4}{|c|}{0.9375} \\
\hline & kappa & \multicolumn{4}{|c|}{0.7547} \\
\hline
\end{tabular}

kernel functions and assigned initial values for them. We tried to perform four types of kernel functions, including radial basis function (RBF_kernel), wavelet kernel function (Wav_kernel), linear kernel function (Lin_kernel), and polynomial kernel function (Poly_kernel). Table 3 summarized the detailed results of classification performance in the $\mathrm{PD}$ dataset in terms of ACC, sensitivity, specificity, AUC, $f$ measure, and KS value, and these results were achieved via 10 -fold CV scheme and represented in the form of average accuracy (Mean), standard deviation (SD), maximal accuracy (Max), and minimal accuracy (Min). From this table, it can be seen that the classification performance of KELM with various kernel functions is apparently differential. The best kernel function of KELM classifier in discriminating the PD dataset was RBF kernel function. We can see that KELM with RBF kernel outperforms that with the other three kernel functions with a mean accuracy of $95.89 \%, 96.35 \%, 95.72 \%$, and $96.04 \%$ in terms of ACC, sensitivity, specificity, and AUC and has also got $f$-measure value of 0.9724 and KS value of 0.8925 . KELM with wavelet kernel has obtained the average results of $94.36 \%, 91.24 \%, 95.25 \%$, and $93.19 \%$ in terms of ACC, sensitivity, specificity, and AUC and got $f$-measure value of 0.9622 and KS value of 0.8425 , lower than those of KELM with RBF kernel. The worse results of classification
TABLE 4: The cluster centers of the features of PD dataset using SCFW method.

\begin{tabular}{|c|c|c|}
\hline $\begin{array}{l}\text { Number of } \\
\text { feature }\end{array}$ & $\begin{array}{c}\text { Centers of the } \\
\text { features using SCFW } \\
\text { (normal case) }\end{array}$ & $\begin{array}{l}\text { Centers of the } \\
\text { features using } \\
\text { SCFW (PD case) }\end{array}$ \\
\hline $\mathrm{F} 1$ & 154.229 & 181.938 \\
\hline $\mathrm{F} 2$ & 197.105 & 223.637 \\
\hline F3 & 116.325 & 145.207 \\
\hline $\mathrm{F} 4$ & 0.006 & 0.006 \\
\hline F5 & 0 & 0 \\
\hline F6 & 0.003 & 0.003 \\
\hline F7 & 0.003 & 0.003 \\
\hline F8 & 0.01 & 0.01 \\
\hline F9 & 0.03 & 0.03 \\
\hline F10 & 0.282 & 0.276 \\
\hline F11 & 0.016 & 0.015 \\
\hline F12 & 0.018 & 0.018 \\
\hline F13 & 0.024 & 0.013 \\
\hline F14 & 0.047 & 0.045 \\
\hline F15 & 0.025 & 0.028 \\
\hline F16 & 21.886 & 24.678 \\
\hline F17 & 0.499 & 0.443 \\
\hline F18 & 0.718 & 0.696 \\
\hline F19 & -5.684 & -6.759 \\
\hline F20 & 0.227 & 0.161 \\
\hline $\mathrm{F} 21$ & 2.382 & 2.155 \\
\hline F22 & 0.207 & 0.123 \\
\hline
\end{tabular}

performance obtained by KELM with polynomial kernel and KELM with linear kernel were successively given. Noting training KELM with kernel functions instead of sigmoid additive function of ELM, the number of hidden neurons has no influence on the performance of KELM model, so it does not need to be considered.

To investigate whether SCFW method can improve the performance of KELM, we further conducted the model in the PD dataset in the weighted feature space by SCFW. The proposed system consisted of two stages. Firstly, SCFW approach was used to weight the features of PD dataset. By using SCFW method, the weighted feature space was constructed. Table 4 listed the cluster centers of the features in the PD dataset using SCFW method. Figure 4 depicted the box graph representation of the original and weighted PD dataset with the whole 22 features. Figure 5 showed the distribution of two classes of the original and weighted 195 samples formed by the best three principle components obtained with the principle component analysis (PCA) algorithm [31]. From Figures 4 and 5, it can be seen that the discriminative ability of the original PD dataset has been improved substantially by SCFW approach. After data preprocessing stage, the classification algorithms have been used and discriminated the weighted PD dataset. 


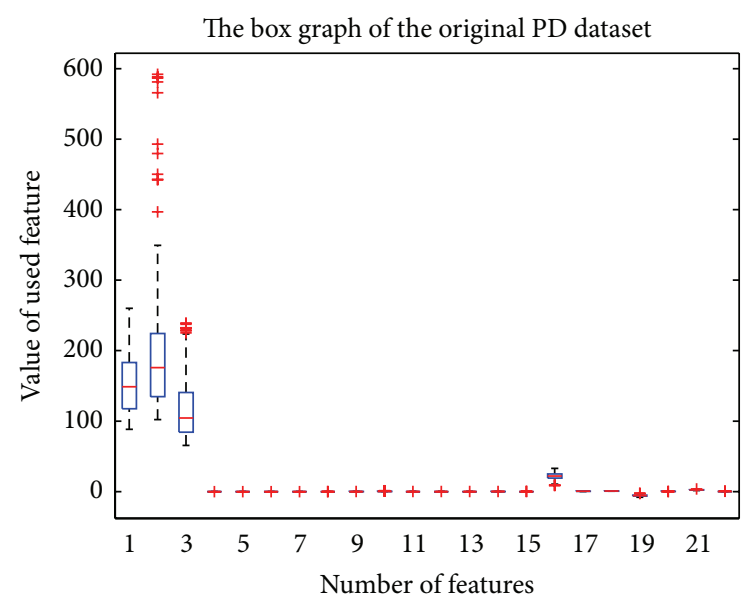

(a)

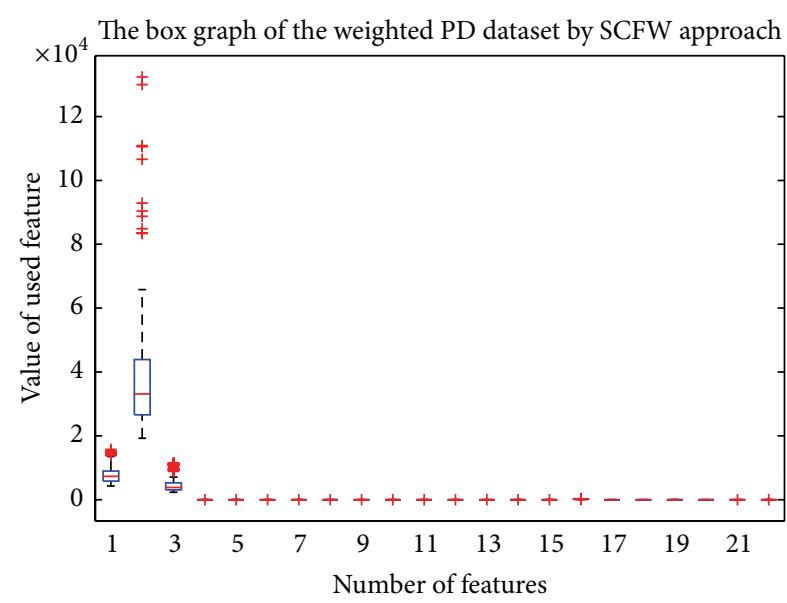

(b)

FIGURE 4: The box graph representation of the original and weighted PD dataset.

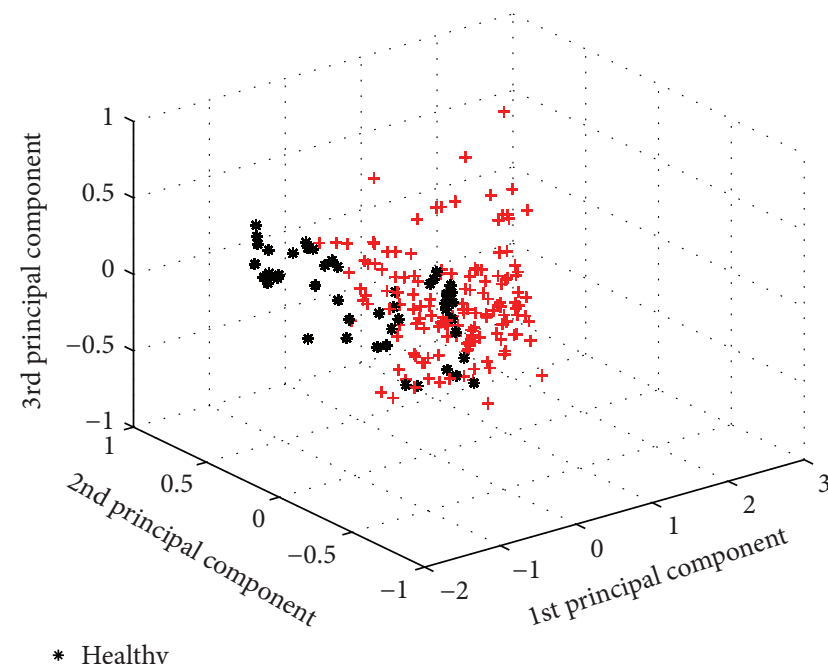

$*$ Heath
$+\mathrm{PD}$

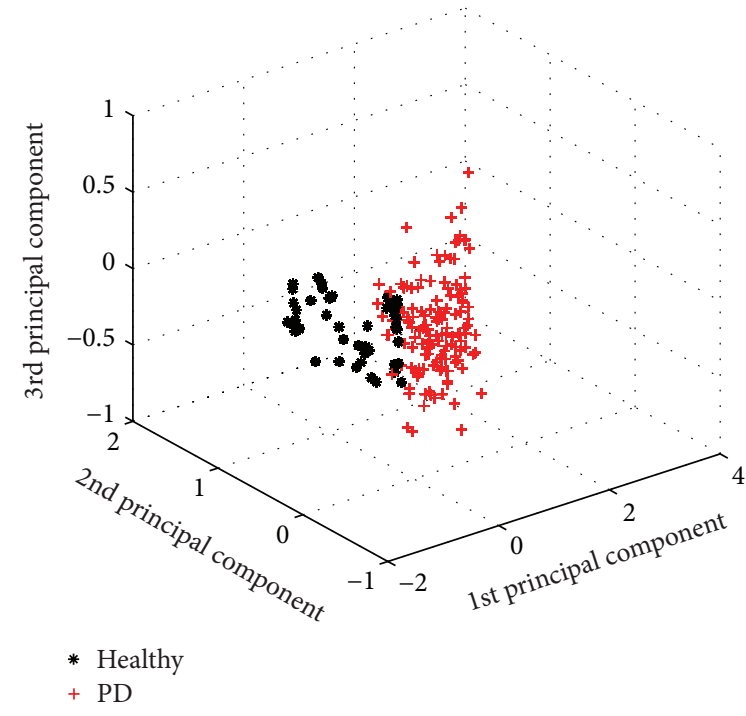

(b)

FIGURE 5: Three-dimensional distribution of two classes in the original and weighted feature space by the best three principle components obtained with PCA method.

The detailed results obtained by SCFW-KELM with four types of different kernel functions were presented in Table 5. As seen from Table 5, all these best results were much higher than the ones obtained in the original feature space without SCFW. The classification performance in the PD dataset has significantly improved by using SCFW method. Compared with KELM with RBF kernel function in the original feature space, KELM with RBF kernel based on SCFW method increased the performance by $3.6 \%, 3.65 \%, 3.67 \%$, and $3.65 \%$ in terms of ACC, sensitivity, specificity, and AUC and has obtained highest $f$-measure value of 0.9966 and highest KS value of 0.9863 . The KELM models with the other three kernel functions also have got great improvements in terms of six performance metrics.
Table 6 also presented the comparison results of the confusion matrices obtained by SCFW-KELM and KELM. As seen from Table 6, SFCW-KELM correctly classified 194 normal cases out of 195 total normal cases and misclassified only one patient with PD as a healthy person, while KELM without SCFW method only correctly classified 187 normal cases out of 195 total normal cases and misclassified 6 patients with $\mathrm{PD}$ as healthy persons and 2 healthy persons as patients with PD.

For SVM classifier, we have performed SVM classifier with RBF kernel. It is known that the performance of SVM is sensitive to the combination of the penalty parameter $C$ and the kernel parameter $\gamma$. Thus, the best combination of $(C, \gamma)$ 
TABLE 5: Results of SCFW-KELM with different types of kernel functions in the PD dataset.

\begin{tabular}{|c|c|c|c|c|c|}
\hline Kernel type & $\begin{array}{c}\text { Performance } \\
\text { metrics }\end{array}$ & Mean & SD & Max & Min \\
\hline \multirow{6}{*}{ RBF_kernel } & $\operatorname{ACC}(\%)$ & 99.49 & 1.15 & 100 & 97.44 \\
\hline & Sensitivity (\%) & 100 & 0 & 100 & 100 \\
\hline & Specificity (\%) & 99.39 & 1.36 & 100 & 96.97 \\
\hline & AUC (\%) & 99.69 & 0.68 & 100 & 98.48 \\
\hline & $f$-measure & \multicolumn{4}{|c|}{0.9966} \\
\hline & Kappa & \multicolumn{4}{|c|}{0.9863} \\
\hline \multirow{6}{*}{ Wav_kernel } & $\operatorname{ACC}(\%)$ & 96.92 & 2.15 & 100 & 94.87 \\
\hline & Sensitivity (\%) & 98.46 & 3.44 & 100 & 92.31 \\
\hline & Specificity (\%) & 96.54 & 2.39 & 100 & 93.33 \\
\hline & AUC (\%) & 97.50 & 2.18 & 100 & 94.23 \\
\hline & $f$-measure & \multicolumn{4}{|c|}{0.9793} \\
\hline & Kappa & \multicolumn{4}{|c|}{0.9194} \\
\hline \multirow{6}{*}{ Lin_kernel } & $\operatorname{ACC}(\%)$ & 96.92 & 2.15 & 100 & 94.87 \\
\hline & Sensitivity (\%) & 90.43 & 8.85 & 100 & 81.82 \\
\hline & Specificity (\%) & 99.29 & 1.60 & 100 & 96.43 \\
\hline & AUC (\%) & 94.86 & 3.99 & 100 & 90.91 \\
\hline & $f$-measure & \multicolumn{4}{|c|}{0.9798} \\
\hline & Kappa & \multicolumn{4}{|c|}{0.9147} \\
\hline \multirow{6}{*}{ Poly_kernel } & $\operatorname{ACC}(\%)$ & 97.43 & 2.56 & 100 & 94.87 \\
\hline & Sensitivity (\%) & 96.67 & 7.45 & 100 & 83.33 \\
\hline & Specificity (\%) & 97.37 & 3.61 & 100 & 93.10 \\
\hline & AUC (\%) & 97.02 & 3.42 & 100 & 91.67 \\
\hline & $f$-measure & \multicolumn{4}{|c|}{0.9828} \\
\hline & Kappa & \multicolumn{4}{|c|}{0.9323} \\
\hline
\end{tabular}

TABLE 6: Confusion matrix of KELM with RBF kernel function in the original and weighted PD dataset.

\begin{tabular}{lccc}
\hline Method & Expected output & \multicolumn{2}{c}{ Prediction output } \\
\hline \multirow{2}{*}{ KELM } & Patients with PD & 141 & 6 \\
& Healthy persons & 2 & 46 \\
\hline \multirow{2}{*}{ SCFW-KELM } & Patients with PD & 146 & 1 \\
& Healthy persons & 0 & 48 \\
\hline
\end{tabular}

needs to select in the classification tasks. Instead of manually setting the parameters $(C, \gamma)$ of SVM, the grid-search technique [32] was adopted using 10 -fold CV to find out the best parameter values. The range of the related parameters $C$ and $\gamma$ was varied between $C=\left[2^{-15}, 2^{-14}, \ldots, 2^{11}\right]$ and $\gamma=\left[2^{-15}, 2^{-14}, \ldots, 2^{5}\right]$. The combinations of $(C, \gamma)$ were tried and the one with the best classification accuracy was chosen as the parameter values of RBF kernel for training model.

For original ELM, we know that the classification performance of ELM with sigmoid additive function is sensitive to the number of hidden neurons $L$, so value of $L$ needs to be specified by users. Figure 6 presented the detailed results of ELM in the original and weighted PD dataset with different hidden neurons ranging from 1 to 50 . Specifically, the average results of 10 runs of 10 -fold CV for every specified neuron were recorded. As shown in Figure 6, the classification rates of ELM were improved with hidden neuron increasing at first and then gradually fluctuated. In the original dataset, it achieved highest mean classification accuracy with 40 hidden neurons, while in the weighted dataset with SCFW method, highest mean classification accuracy was gained with only 26 hidden neurons.

For KNN classifier, the influence of neighborhood size $k$ of $\mathrm{KNN}$ classifier in the classification performance of the PD dataset has been investigated. In this study, value of $k$ increased from 1 to 10 . The results obtained from $\mathrm{KNN}$ classifier with different values of $k$ in the PD dataset are shown in Figure 7. From the figure, we can see that the best results have been obtained by 1-NN classifier, and the performance was decreased with the value of $k$ increasing, while the better results were achieved in the weighted PD dataset with SCFW method for 2-NN.

For KELM classifier, there were two parameters, the penalty parameter $C$ and the kernel parameter $\gamma$, that need to be specified. In this study, we have conducted the experiments on KELM depending on the best combination of $(C, \gamma)$ by grid-search strategy. The parameters $C$ and $\gamma$ were both varied in the range of $\left[2^{-15}, 2^{-14}, \ldots, 2^{15}\right]$ with the step size of 1 . Figure 8 showed the classification accuracy surface in one run of 10 -fold CV procedure, where $x$-axis and $y$-axis were $\log _{2} C$ and $\log _{2} \gamma$, respectively. Each mesh node in the $(x, y)$ plane of the classification accuracy represented a parameter combination and $z$-axis denoted the achieved test accuracy value with each parameter combination.

Table 7 summarized the comprehensive results achieved from four classifiers and those based on SCFW method in terms of ACC, sensitivity, specificity, AUC, $f$-measure, and KS value over 10 runs of 10 -fold CV. Besides, the sum of computational time of training and that of testing in seconds was recorded. In this table, we can see that, with the aid of SCFW method, all these best results were much higher than the ones obtained in the original feature space. The SCFWKELM model has achieved highest results of $99.49 \%, 100 \%$, $99.39 \%$, and $99.69 \%$ in terms of ACC, sensitivity, specificity, and AUC and got highest $f$-measure of 0.9966 and KS value of 0.9863 , which outperforms the other three algorithms. Compared with KELM without SCFW, SCFW-KELM has improved the average performance by $3.6 \%, 3.65 \%, 3.67 \%$, and $3.65 \%$ in terms of ACC, sensitivity, specificity, and AUC. Note that the running time of SCFW-KELM was extremely short, which costs only 0.0126 seconds.

In comparison with SVM, SCFW-SVM has achieved the results of $97.95 \%, 96.67 \%, 98.71 \%$, and $97.6 \%$ in terms of ACC, sensitivity, specificity, and AUC and improved the performance by $2.57 \%, 11.58 \%, 0.04 \%$, and $5.72 \%$, respectively. KNN also has significantly improved by SCFW method. For ELM classifier, it has achieved best results by ELM with 36 hidden neurons on the original feature space, while the best performance was achieved by SCFW-ELM with small hidden neurons (only 26). It meant that the combination of SCFW 


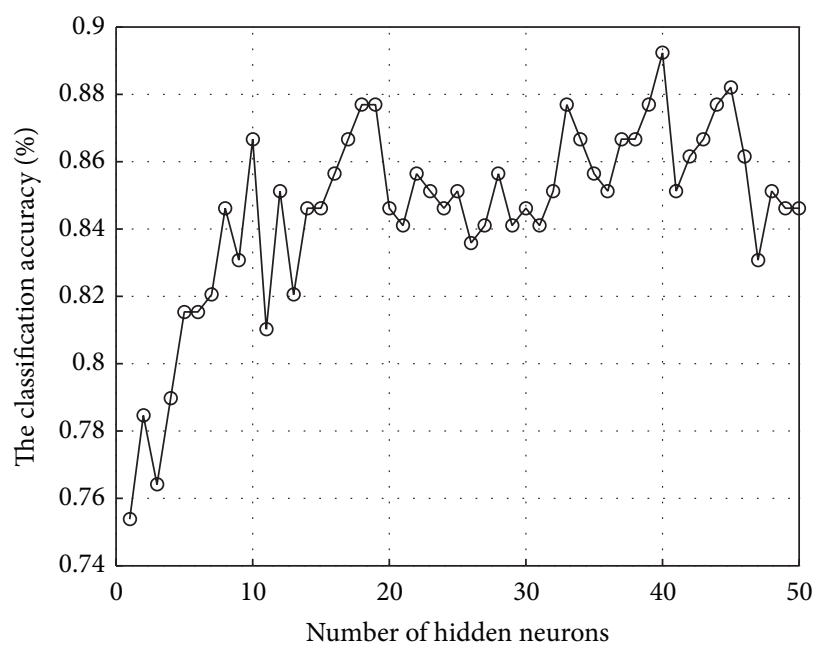

(a) Effects of $L$ in ELM in original dataset

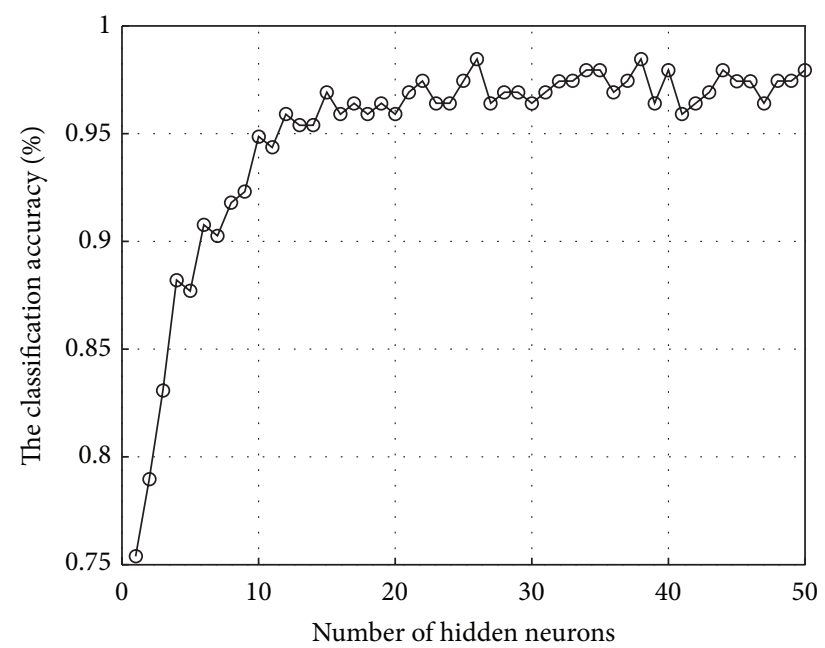

(b) Effects of $L$ in ELM in weighted dataset

FIgURE 6: The effects of hidden neurons in original ELM in the classification of the original and weighted PD dataset.

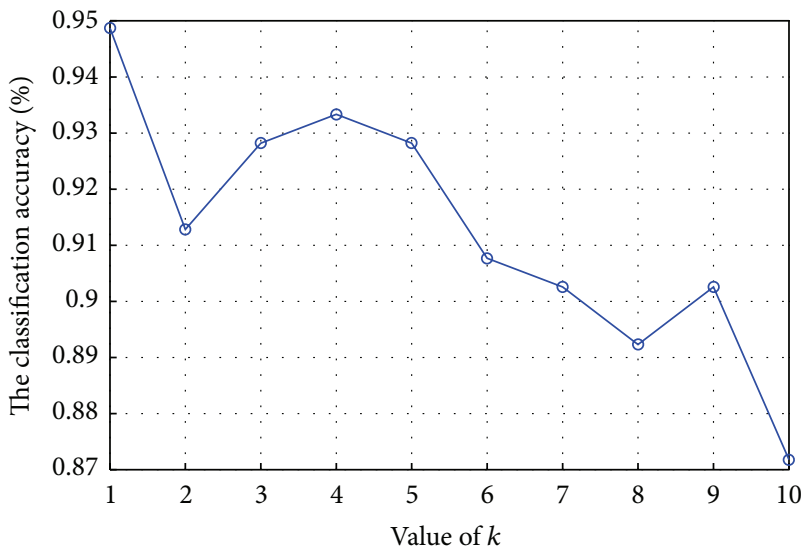

(a) Effects of $k$ in $\mathrm{KNN}$ in original dataset

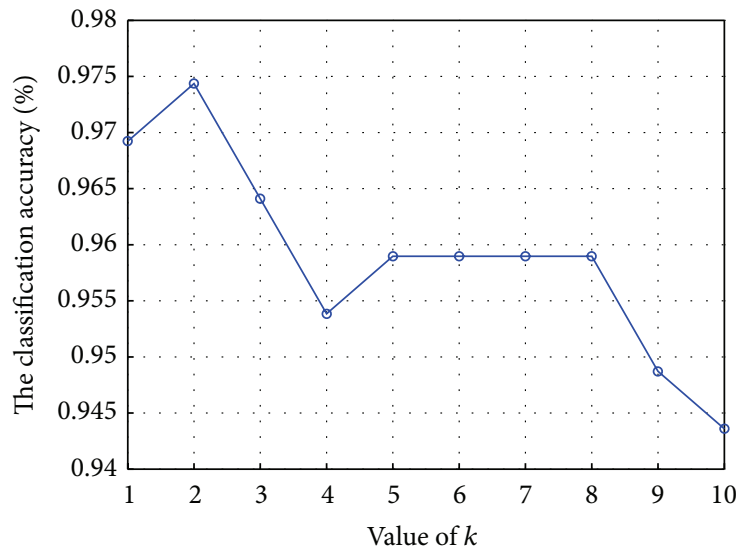

(b) Effects of $k$ in $\mathrm{KNN}$ in weighted dataset

FIGURE 7: The effects of $k$ in KNN in the classification of the original and weighted PD dataset.

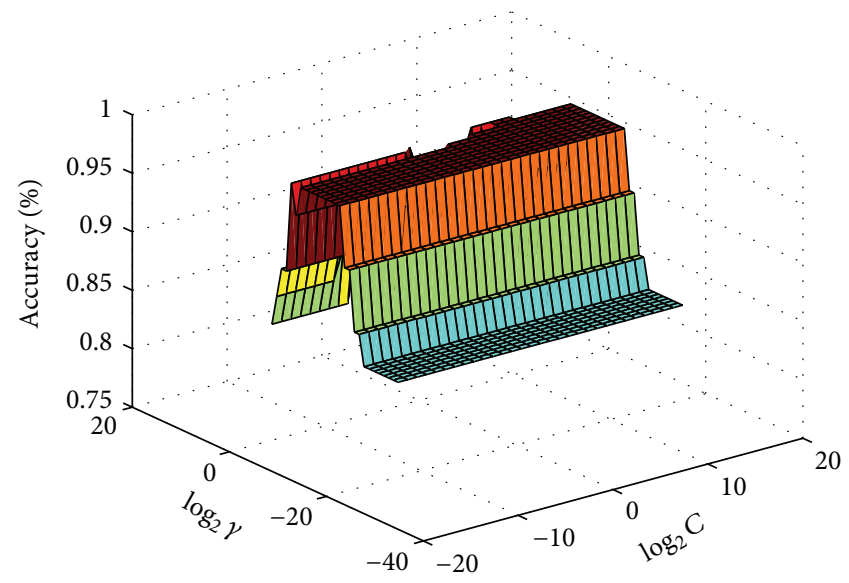

(a) Effects of $(C, \gamma)$ in original dataset

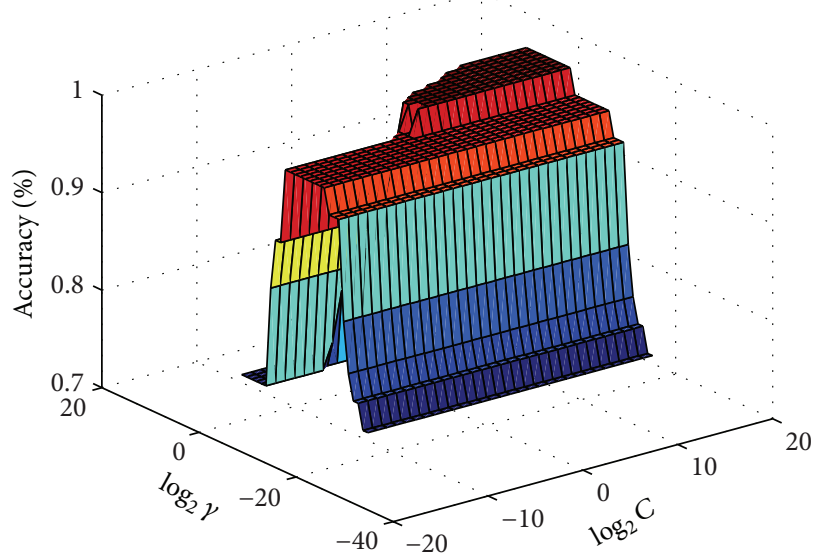

(b) Effects of $(C, \gamma)$ in weighted dataset

FIGURE 8: Test accuracy surface with parameters in KELM in the original and weighted PD dataset. 
TABLE 7: The results obtained from four algorithms in the original and weighted PD dataset.

\begin{tabular}{|c|c|c|c|}
\hline Methods & $\begin{array}{l}\text { Performance } \\
\text { metrics }\end{array}$ & $\begin{array}{l}\text { Original feature } \\
\text { space without } \\
\text { SCFW method }\end{array}$ & $\begin{array}{c}\text { Weighted } \\
\text { feature space } \\
\text { with SCFW } \\
\text { method }\end{array}$ \\
\hline \multirow{7}{*}{ KELM-RBF } & $\operatorname{ACC}(\%)$ & $95.89 \pm 4.66$ & $99.49 \pm 1.15$ \\
\hline & Sensitivity (\%) & $96.35 \pm 5.19$ & $100 \pm 0$ \\
\hline & Specificity (\%) & $95.72 \pm 5.93$ & $99.39 \pm 1.36$ \\
\hline & AUC (\%) & $96.04 \pm 4.06$ & $99.69 \pm 0.68$ \\
\hline & $f$-measure & 0.9724 & 0.9966 \\
\hline & Kappa & 0.8925 & 0.9863 \\
\hline & Time (s) & 0.00435 & 0.0126 \\
\hline \multirow{7}{*}{ SVM } & $\operatorname{ACC}(\%)$ & $95.38 \pm 1.15$ & $97.95 \pm 2.15$ \\
\hline & Sensitivity (\%) & $85.09 \pm 10.45$ & $96.67 \pm 7.45$ \\
\hline & Specificity (\%) & $98.67 \pm 2.98$ & $98.71 \pm 1.77$ \\
\hline & AUC (\%) & $91.88 \pm 4.14$ & $97.69 \pm 3.46$ \\
\hline & $f$-measure & 0.9699 & 0.9863 \\
\hline & Kappa & 0.8711 & 0.9447 \\
\hline & Time (s) & 1.24486 & 1.29817 \\
\hline \multirow{7}{*}{ KNN } & ACC (\%) & $95.38 \pm 5.25$ & $97.43 \pm 3.14$ \\
\hline & Sensitivity (\%) & $92.73 \pm 11.85$ & $97.78 \pm 4.97$ \\
\hline & Specificity (\%) & $96.50 \pm 4.38$ & $97.38 \pm 4.10$ \\
\hline & AUC (\%) & $94.61 \pm 6.95$ & $97.58 \pm 2.60$ \\
\hline & $f$-measure & 0.9692 & 0.9828 \\
\hline & Kappa & 0.8765 & 0.9431 \\
\hline & Time (s) & 1.2847 & 1.3226 \\
\hline \multirow{7}{*}{ ELM } & $\mathrm{ACC}(\%)$ & $89.23 \pm 6.88$ & $96.92 \pm 4.21$ \\
\hline & Sensitivity (\%) & $73.94 \pm 13.18$ & $95.78 \pm 5.79$ \\
\hline & Specificity (\%) & $93.35 \pm 6.27$ & $97.19 \pm 4.51$ \\
\hline & AUC (\%) & $83.64 \pm 9.06$ & $96.48 \pm 4.36$ \\
\hline & $f$-measure & $83.64 \pm 9.06$ & 0.9863 \\
\hline & Kappa & 0.7078 & 0.9447 \\
\hline & Time (s) & 1.1437 & 1.2207 \\
\hline
\end{tabular}

and ELM not only significantly improved the performance but also compacted the network structure of ELM. Moreover, the sensitive results of SVM and ELM were significantly improved by $11.58 \%$ and $21.84 \%$, respectively. Whatever in the original or weighted feature space, KELM with RBF kernel was much superior to the other three models by a large percentage in terms of ACC, sensitivity, specificity, AUC, $f$-measure, and KS value. Although SVM achieved the specificity of $98.67 \%$, the sensitivity, AUC, $f$-measure, and KS value were lower than those of KELM with RBF kernel. We can also see that the performance of KELM with RBF kernel was much higher than those of ELM with sigmoid function. The reason may lie in the fact that the relation between class labels and features in the PD dataset is linearly nonseparable; kernel-based strategy works better for this case by transforming from linearly nonseparable to linearly separable dataset. However, the performances obtained by
SCFW-SVM approach were close to those of SCFW-KNN. It meant that, after data preprocessing, SVM can achieve the same ability to discriminate the PD dataset as that of KNN.

Additionally, it is interesting to find that the standard deviation of SCFW-KELM was much lower than that of KELM, and it had the smallest SD in all of the models, which meant SCFW-KELM became more robust and reliable by means of SCFW method. In addition, the reason why SCFW method outperforms FCM is that SCFW may be more suitable for nonlinear separable datasets. It considers the density measure of data points to reduce the influence of outliers; however, FCM tends to select outliers as initial centers.

For comparison purpose, the classification accuracies achieved by previous methods which researched the PD diagnosis problem were presented in Table 8 . As shown in the table, our developed method can obtain better classification results than all available methods proposed in previous studies.

Experiment 2 (classification in two other benchmark datasets). Besides the PD dataset, two benchmark datasets, that is, Cleveland Heart and Wisconsin Diagnostic Breast Cancer (WDBC) datasets, from the UCI machine learning repository, have been used to further evaluate the efficiency and effectiveness of the proposed method. We used the same flow as in the PD dataset for the experiments of two datasets. The weighted features space of datasets was constructed using SCFW and then the weighted features were evaluated with the four mentioned algorithms. It will only give the classification results of four algorithms for the sake of convenience. Table 9 showed the obtained results in the classification of the original and weighted Cleveland Heart dataset by SCFWKELM model. Table 10 presented the achieved results in the classification of the original and weighted WDBC dataset using SCFW-KELM model. As seen from these results, the proposed method also has achieved excellent results. It indicated the generality of the proposed method.

\section{Conclusions and Future Work}

In this work, we have developed a new hybrid diagnosis method for addressing the PD problem. The main novelty of this paper lies in the proposed approach; the combination of SCFW method and KELM with different types of kernel functions allows the detection of PD in an efficient and fast manner. Experiments results have demonstrated that the proposed system performed significantly well in discriminating the patients with PD and healthy ones. Meanwhile, the comparative results are conducted among KELM, SVM, KNN, and ELM. The experiment results have shown that the SCFW-KELM method performs advantageously over the other three methods in terms of ACC, sensitivity, specificity, AUC, $f$-measure, and kappa statistic value. In addition, the proposed system outperforms the existing methods proposed in the literature. Based on the empirical analysis, it indicates that the proposed method can be used as a promising alternative tool in medical decision making for PD diagnosis. 
TABLE 8: Classification accuracies achieved with our method and other methods.

\begin{tabular}{lll}
\hline Study & Method & Accuracy (\%) \\
\hline Little et al. [6] & Preselection filter + exhaustive search + SVM & 91.40 (bootstrap with 50 replicates) \\
Shahbaba and Neal [7] & Dirichlet process mixtures & 87.70 (5-fold CV) \\
Das [8] & ANN & 92.90 (hold out) \\
Sakar and Kursun [9] & Mutual information + SVM & 92.75 (bootstrap with 50 replicates) \\
Psorakis et al. [10] & Improved mRVMs & 89.47 (10-fold CV) \\
Guo et al. [11] & GP-EM & 93.10 (10-fold CV) \\
Luukka [12] & Fuzzy entropy measures + similarity & 85.03 (hold out) \\
Ozcift and Gulten [14] & CFS-RF & 87.10 (10-fold CV) \\
Li et al. [13] & Fuzzy-based nonlinear transformation + SVM & 93.47 (hold out) \\
Åström and Koker [15] & Parallel NN & 91.20 (hold out) \\
Spadoto et al. [16] & PSO + OPF Harmony search + OPF Gravitational search + OPF & 73.53 (hold out) 84.01 (hold out) 84.01 (hold out) \\
Daliri [19] & SVM with chi-square distance kernel & 91.20 (50-50\% training-testing) \\
Polat [17] & FCMFW + KNN & 97.93 (50-50\% training-testing) \\
Chen et al. [18] & PCA-FKNN & 96.07 (average 10-fold CV) \\
Zuo et al. [20] & PSO-FKNN & 97.47 (10-fold CV) \\
This study & SCFW-KELM & $\mathbf{9 9 . 4 9 ~ ( 1 0 - f o l d ~ C V ) ~}$ \\
\hline
\end{tabular}

TABLE 9: Results of SCFW-KELM with different types of kernel functions in Cleveland heart dataset.

\begin{tabular}{|c|c|c|c|c|c|}
\hline Kernel type & $\begin{array}{l}\text { Performance } \\
\text { metrics }\end{array}$ & Mean & SD & Max & Min \\
\hline \multirow{6}{*}{ RBF_kernel } & $\operatorname{ACC}(\%)$ & 99.34 & 0.91 & 100 & 98.33 \\
\hline & Sensitivity (\%) & 100 & 0 & 100 & 100 \\
\hline & Specificity (\%) & 98.75 & 1.72 & 100 & 96.67 \\
\hline & AUC (\%) & 99.37 & 0.86 & 100 & 98.33 \\
\hline & $f$-measure & \multicolumn{4}{|c|}{0.9964} \\
\hline & Kappa & \multicolumn{4}{|c|}{0.9867} \\
\hline \multirow{6}{*}{ Wav_kernel } & ACC (\%) & 99.01 & 0.90 & 100 & 98.36 \\
\hline & Sensitivity (\%) & 100 & 0 & 100 & 100 \\
\hline & Specificity (\%) & 97.84 & 2.02 & 100 & 95.83 \\
\hline & AUC (\%) & 98.92 & 1.01 & 100 & 97.92 \\
\hline & $f$-measure & \multicolumn{4}{|c|}{0.9891} \\
\hline & Kappa & \multicolumn{4}{|c|}{0.98} \\
\hline \multirow{6}{*}{ Lin_kernel } & ACC (\%) & 93.07 & 93.07 & 93.07 & 93.07 \\
\hline & Sensitivity (\%) & 98.77 & 98.77 & 98.77 & 98.77 \\
\hline & Specificity (\%) & 87.05 & 87.05 & 87.05 & 87.05 \\
\hline & AUC (\%) & 92.91 & 92.91 & 92.91 & 92.91 \\
\hline & $f$-measure & \multicolumn{4}{|c|}{0.9195} \\
\hline & Kappa & \multicolumn{4}{|c|}{0.8591} \\
\hline \multirow{6}{*}{ Poly_kernel } & $\operatorname{ACC}(\%)$ & 98.35 & 2.33 & 100 & 95.08 \\
\hline & Sensitivity (\%) & 100 & 0 & 100 & 100 \\
\hline & Specificity (\%) & 96.60 & 5.01 & 100 & 88.89 \\
\hline & AUC (\%) & 98.30 & 2.50 & 100 & 94.44 \\
\hline & $f$-measure & \multicolumn{4}{|c|}{0.9817} \\
\hline & Kappa & \multicolumn{4}{|c|}{0.9667} \\
\hline
\end{tabular}

TABLE 10: Results of SCFW-KELM with different types of kernel functions in WDBC dataset.

\begin{tabular}{|c|c|c|c|c|c|}
\hline Kernel type & $\begin{array}{c}\text { Performance } \\
\text { metrics }\end{array}$ & Mean & SD & Max & Min \\
\hline \multirow{6}{*}{ RBF_kernel } & $\operatorname{ACC}(\%)$ & 99.65 & 0.79 & 100 & 98.23 \\
\hline & Sensitivity (\%) & 99.05 & 2.13 & 100 & 95.24 \\
\hline & Specificity (\%) & 100 & 0 & 100 & 100 \\
\hline & AUC (\%) & 99.52 & 1.06 & 100 & 97.62 \\
\hline & $f$-measure & \multicolumn{4}{|c|}{0.9972} \\
\hline & Kappa & \multicolumn{4}{|c|}{0.9925} \\
\hline \multirow{6}{*}{ Wav_kernel } & ACC (\%) & 99.65 & 0.48 & 100 & 99.12 \\
\hline & Sensitivity (\%) & 99.10 & 1.24 & 100 & 97.62 \\
\hline & Specificity (\%) & 100 & 0 & 100 & 100 \\
\hline & AUC (\%) & 99.54 & 0.66 & 100 & 98.65 \\
\hline & $f$-measure & \multicolumn{4}{|c|}{0.9958} \\
\hline & Kappa & \multicolumn{4}{|c|}{0.9925} \\
\hline \multirow{6}{*}{ Lin_kernel } & $\operatorname{ACC}(\%)$ & 98.07 & 1.69 & 100 & 95.61 \\
\hline & Sensitivity (\%) & 94.70 & 5.27 & 100 & 86.11 \\
\hline & Specificity (\%) & 100 & 0 & 100 & 100 \\
\hline & AUC (\%) & 97.35 & 2.63 & 100 & 93.06 \\
\hline & $f$-measure & \multicolumn{4}{|c|}{0.9848} \\
\hline & Kappa & \multicolumn{4}{|c|}{0.9582} \\
\hline \multirow{6}{*}{ Poly_kernel } & ACC (\%) & 99.40 & 0.88 & 99.12 & 97.37 \\
\hline & Sensitivity (\%) & 95.33 & 2.07 & 97.73 & 93.48 \\
\hline & Specificity (\%) & 100 & 0 & 100 & 100 \\
\hline & AUC (\%) & 97.67 & 1.04 & 98.86 & 96.74 \\
\hline & $f$-measure & \multicolumn{4}{|c|}{0.9944} \\
\hline & Kappa & \multicolumn{4}{|c|}{0.962} \\
\hline
\end{tabular}


The future investigation will pay much attention to evaluating the proposed method in other medical diagnosis problems.

\section{Conflict of Interests}

The authors declare that there is no conflict of interests regarding the publication of this paper.

\section{Acknowledgments}

This research is supported by the Natural Science Foundation of China (NSFC) under Grant nos. 61170092, 61133011, 61272208, 61103091, 61202308, and 61303113. This research is also supported by the open project program of Key Laboratory of Symbolic Computation and Knowledge Engineering of Ministry of Education, Jilin University, under Grant no. 93K172013K01.

\section{References}

[1] G. F. Wooten, L. J. Currie, V. E. Bovbjerg, J. K. Lee, and J. Patrie, "Are men at greater risk for Parkinson's disease than women?" Journal of Neurology, Neurosurgery \& Psychiatry, vol. 75, no. 4, pp. 637-639, 2004.

[2] K. R. Chaudhuri, D. G. Healy, and A. H. V. Schapira, "Nonmotor symptoms of Parkinson's disease: diagnosis and management," The Lancet Neurology, vol. 5, no. 3, pp. 235-245, 2006.

[3] A. K. Ho, R. Iansek, C. Marigliani, J. L. Bradshaw, and S. Gates, "Speech impairment in a large sample of patients with Parkinson's disease," Behavioural Neurology, vol. 11, no. 3, pp. 131-137, 1998.

[4] K. M. Rosen, R. D. Kent, A. L. Delaney, and J. R. Duffy, "Parametric quantitative acoustic analysis of conversation produced by speakers with dysarthria and healthy speakers," Journal of Speech, Language, and Hearing Research, vol. 49, no. 2, pp. 395411, 2006.

[5] D. A. Rahn III, M. Chou, J. J. Jiang, and Y. Zhang, "Phonatory impairment in Parkinson's disease: evidence from nonlinear dynamic analysis and perturbation analysis," Journal of Voice, vol. 21, no. 1, pp. 64-71, 2007.

[6] M. A. Little, P. E. McSharry, E. J. Hunter, J. Spielman, and L. O. Ramig, "Suitability of dysphonia measurements for telemonitoring of Parkinson's disease," IEEE Transactions on Biomedical Engineering, vol. 56, no. 4, pp. 1015-1022, 2009.

[7] B. Shahbaba and R. Neal, "Nonlinear models using Dirichlet process mixtures," Journal of Machine Learning Research, vol. 10, pp. 1829-1850, 2009.

[8] R. Das, "A comparison of multiple classification methods for diagnosis of Parkinson disease," Expert Systems with Applications, vol. 37, no. 2, pp. 1568-1572, 2010.

[9] C. O. Sakar and O. Kursun, “Telediagnosis of parkinson’s disease using measurements of dysphonia," Journal of Medical Systems, vol. 34, no. 4, pp. 591-599, 2010.

[10] I. Psorakis, T. Damoulas, and M. A. Girolami, "Multiclass relevance vector machines: sparsity and accuracy," IEEE Transactions on Neural Networks, vol. 21, no. 10, pp. 1588-1598, 2010.

[11] P.-F. Guo, P. Bhattacharya, and N. Kharma, "Advances in detecting Parkinson's disease," in Medical Biometrics, vol. 6165 of Lecture Notes in Computer Science, pp. 306-314, Springer, Berlin, Germany, 2010.
[12] P. Luukka, "Feature selection using fuzzy entropy measures with similarity classifier," Expert Systems with Applications, vol. 38, no. 4, pp. 4600-4607, 2011.

[13] D.-C. Li, C.-W. Liu, and S. C. Hu, "A fuzzy-based data transformation for feature extraction to increase classification performance with small medical data sets," Artificial Intelligence in Medicine, vol. 52, no. 1, pp. 45-52, 2011.

[14] A. Ozcift and A. Gulten, "Classifier ensemble construction with rotation forest to improve medical diagnosis performance of machine learning algorithms," Computer Methods and Programs in Biomedicine, vol. 104, no. 3, pp. 443-451, 2011.

[15] F. Åström and R. Koker, "A parallel neural network approach to prediction of Parkinson's Disease," Expert Systems with Applications, vol. 38, no. 10, pp. 12470-12474, 2011.

[16] A. A. Spadoto, R. C. Guido, F. L. Carnevali, A. F. Pagnin, A. X. Falcao, and J. P. Papa, "Improving Parkinson's disease identification through evolutionary-based feature selection," in Proceedings of the Annual International Conference of the IEEE Engineering in Medicine and Biology Society (EMBC '11), pp. 7857-7860, Boston, Mass, USA, August 2011.

[17] K. Polat, "Classification of Parkinson's disease using feature weighting method on the basis of fuzzy C-means clustering," International Journal of Systems Science, vol. 43, no. 4, pp. 597609, 2012.

[18] H.-L. Chen, C.-C. Huang, X.-G. Yu et al., "An efficient diagnosis system for detection of Parkinson's disease using fuzzy $k$-nearest neighbor approach," Expert Systems with Applications, vol. 40, no. 1, pp. 263-271, 2013.

[19] M. R. Daliri, "Chi-square distance kernel of the gaits for the diagnosis of Parkinson's disease," Biomedical Signal Processing and Control, vol. 8, no. 1, pp. 66-70, 2013.

[20] W.-L. Zuo, Z.-Y. Wang, T. Liu, and H.-L. Chen, "Effective detection of Parkinson's disease using an adaptive fuzzy $k$-nearest neighbor approach," Biomedical Signal Processing and Control, vol. 8, no. 4, pp. 364-373, 2013.

[21] K. Polat and S. S. Durduran, "Subtractive clustering attribute weighting (SCAW) to discriminate the traffic accidents on Konya-Afyonkarahisar highway in Turkey with the help of GIS: a case study," Advances in Engineering Software, vol. 42, no. 7, pp. 491-500, 2011.

[22] K. Polat, "Application of attribute weighting method based on clustering centers to discrimination of linearly non-separable medical datasets," Journal of Medical Systems, vol. 36, no. 4, pp. 2657-2673, 2012.

[23] G.-B. Huang, H. Zhou, X. Ding, and R. Zhang, "Extreme learning machine for regression and multiclass classification," IEEE Transactions on Systems, Man, and Cybernetics Part B: Cybernetics, vol. 42, no. 2, pp. 513-529, 2012.

[24] Q. Y. Zhu, A. K. Qin, P. N. Suganthan, and G. B. Huang, "Evolutionary extreme learning machine," Pattern Recognition, vol. 38, no. 10, pp. 1759-1763, 2005.

[25] S. L. Chiu, "Fuzzy model identification based on cluster estimation," Journal of Intelligent and Fuzzy Systems, vol. 2, no. 3, pp. 267-278, 1994

[26] G.-B. Huang, Q.-Y. Zhu, and C.-K. Siew, "Extreme learning machine: theory and applications," Neurocomputing, vol. 70, no. 1-3, pp. 489-501, 2006.

[27] C.-C. Chang and C.-J. Lin, "LIBSVM: a Library for support vector machines," ACM Transactions on Intelligent Systems and Technology, vol. 2, no. 3, article 27, 2011. 
[28] R. Kohavi, "A study of cross-validation and bootstrap for accuracy estimation and model selection," in Proceedings of the 14th International Joint Conference on Artificial Intelligence (IJCAI '95), pp. 1137-1143, Montreal, Canada, August 1995.

[29] J. Huang and C. X. Ling, "Using AUC and accuracy in evaluating learning algorithms," IEEE Transactions on Knowledge and Data Engineering, vol. 17, no. 3, pp. 299-310, 2005.

[30] A. Ben-David, "Comparison of classification accuracy using Cohen's Weighted Kappa," Expert Systems with Applications, vol. 34, no. 2, pp. 825-832, 2008.

[31] L. I. Smith, A Tutorial on Principal Components Analysis, vol. 51, Cornell University, Ithaca, NY, USA, 2002.

[32] C.-W. Hsu, C.-C. Chang, and C.-J. Lin, A Practical Guide to Support Vector Classification, 2003. 


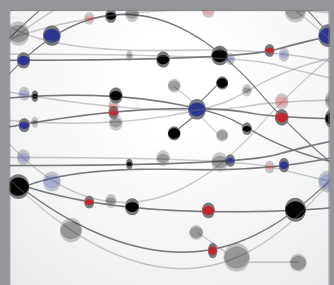

The Scientific World Journal
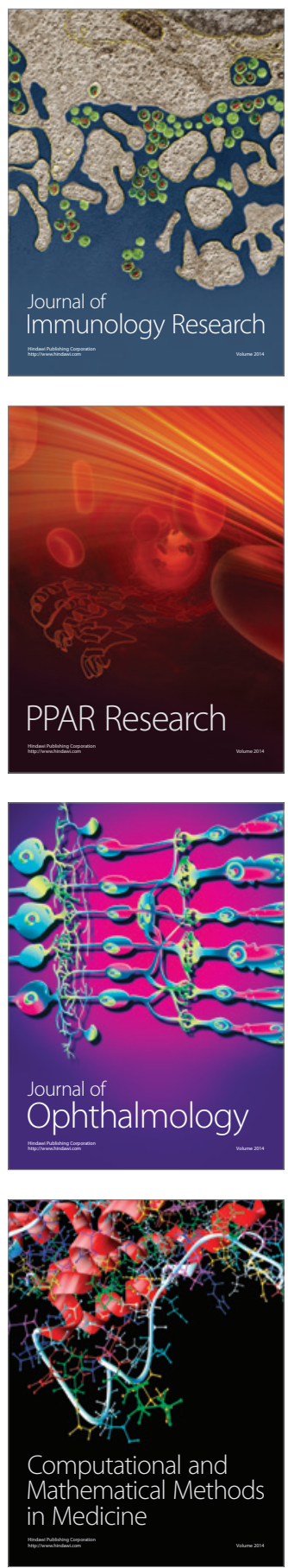

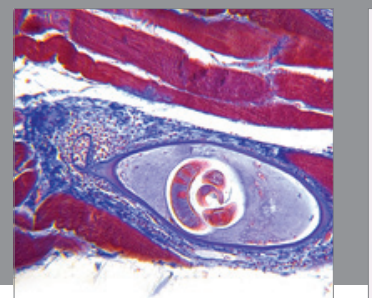

Gastroenterology

Research and Practice
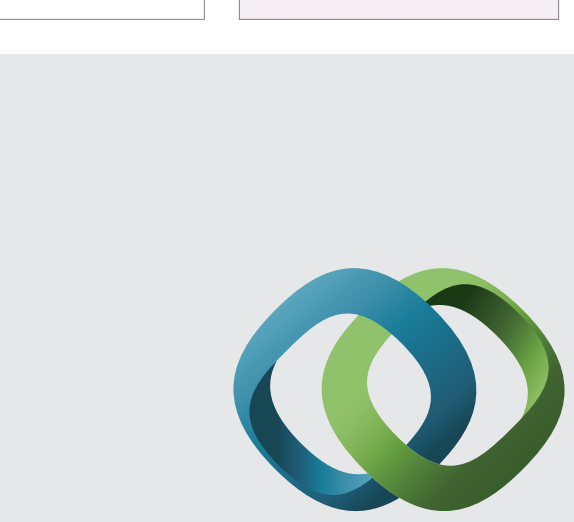

\section{Hindawi}

Submit your manuscripts at

http://www.hindawi.com
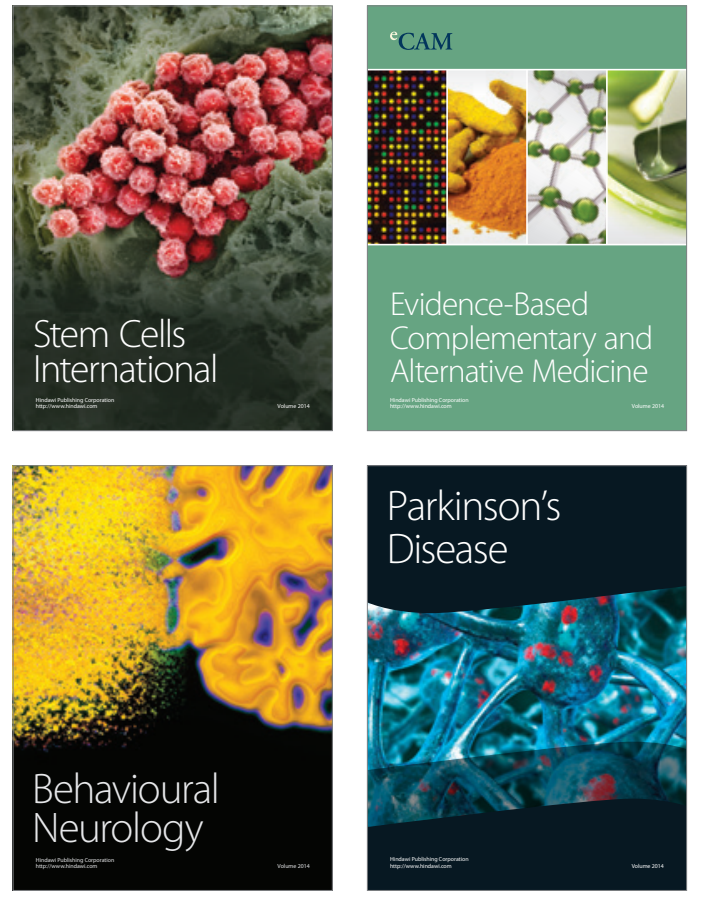
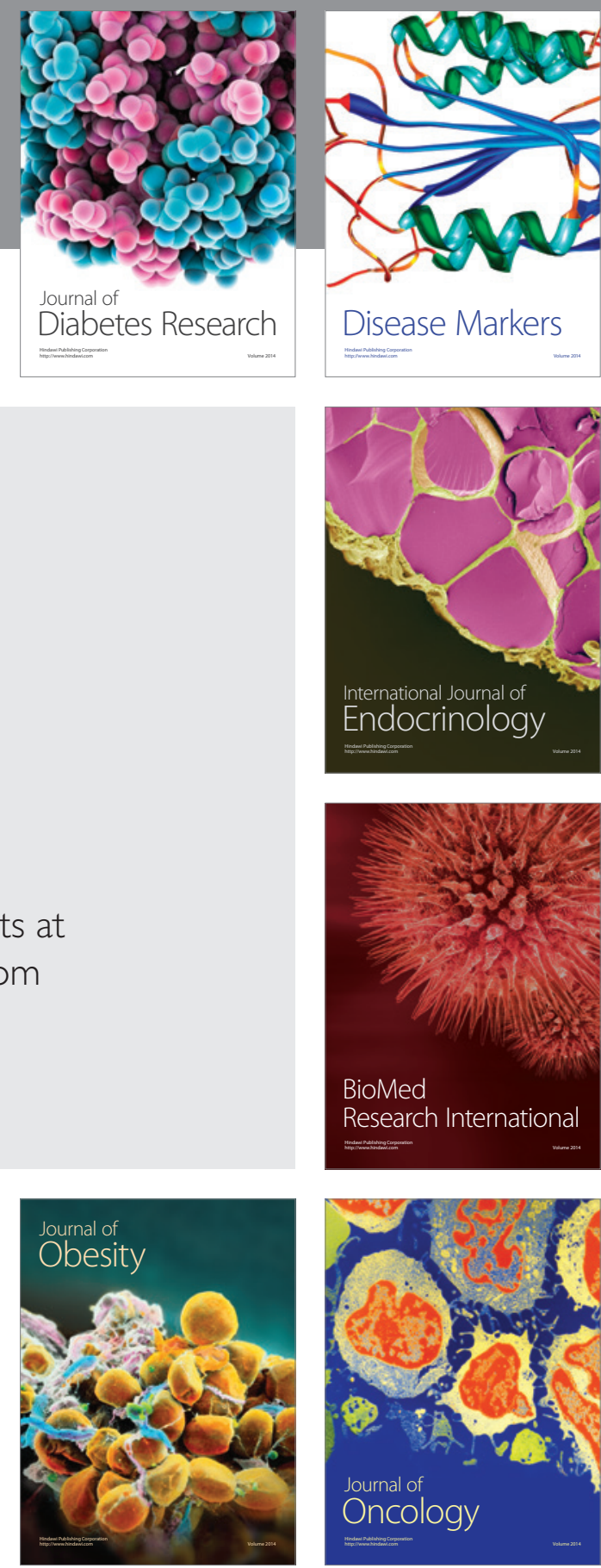

Disease Markers
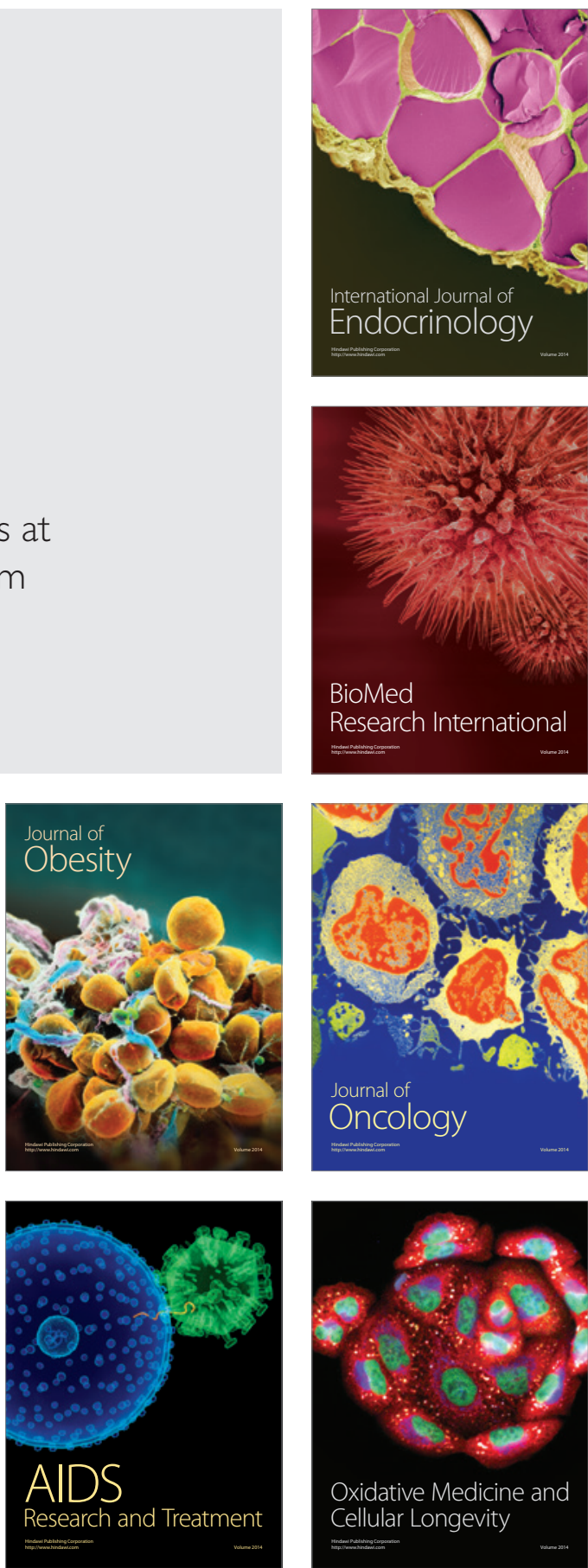